\title{
Levels and Predictors of Self-Care Among Patients with Hypertension in Pakistan
}

This article was published in the following Dove Press journal:

International Journal of General Medicine

\author{
Khairulnissa Ajani ${ }^{1}$ \\ Ambreen Gowani' \\ Raisa Gul ${ }^{1,2}$ \\ Pammla Petrucka ${ }^{3}$ \\ 'School of Nursing, Aga Khan University, \\ Karachi, Pakistan; ${ }^{2}$ School of Nursing, \\ Shifa Tameer-e-Millat University, \\ Islamabad, Pakistan; ${ }^{3}$ College of Nursing, \\ University of Saskatchewan, Saskatoon, \\ Canada
}

Background: Globally, hypertension is the leading non-communicable disease and strongest predictor of cardiovascular diseases. To mitigate and prevent hypertension-related complications, self-care behavior adaptation has proven to be vital. In this study, we examined the six clinically prescribed levels of self-care as prescribed by the Seventh Report of the Joint National Committee on Prevention, Detection, Evaluation and Treatment of High Blood Pressure and its predictors among a select sample of hypertensive individuals in Karachi, Pakistan.

Methods: This study reports the cross-sectional survey of a sequential mixed method study which assessed the levels of self-care of hypertensive individuals residing in an urban cosmopolitan setting within Karachi Pakistan. Four hundred and two patients were screened using the H-SCALE questionnaire, while socio-demographic predictors of self-care and level of knowledge of hypertension were identified using a study-specific checklist. Self-care was assessed against six clinical domains including medication adherence, diet, weight management, physical activity, and abstinence from alcohol.

Results: Participants were recruited from the two largest tertiary care hospitals in Karachi. Good knowledge about hypertension, including its causes, management, and complications was reported by $4.47 \%$ of the participants. Highest levels of self-care adherence were found for abstinence from alcohol (100\%), smoking cessation (83.33\%), and medication compliance $(71.89 \%)$, whereas lowest levels were found for diet $(27.11 \%)$, and physical activity $(24.88 \%)$. In terms of predictors for self-care, age, male gender, and self-checking of blood pressure at home, followed by the level of education were the most common predictors for each self-care behavior in the given population.

Conclusion: Overall knowledge of self-care for hypertension is sub-optimal among hypertensive patients in Pakistan which is reflected in their behaviors. There is a need to introduce healthcare educational programs in Pakistan which can improve self-care behaviors of hypertensive individuals and potentially reduce the prevalence of associated cardiovascular diseases and its complications.

Keywords: hypertension, self-care management, cross-sectional study, Pakistan
Correspondence: Khairulnissa Ajani Aga Khan University School of Nursing, P.O. Box 3500, Stadium Road, Karachi, Pakistan

Email khairulnissa.ajani@aku.edu

\section{Background}

The global demographic shift towards ageing, rapid urbanization, and adoption of unhealthy lifestyle behaviors is significantly affecting trends in human health, generally, ${ }^{1}$ and in the emergence of non-communicable diseases including hypertension, specifically. ${ }^{2}$ Hypertension, a complex and universal health issue, is pervasive within the adult population, bringing an array of associated health issues that may result in a major burden for hypertensive individuals. According to the World 
Health Organization, one in eight deaths globally is caused by hypertension making it the third most common cause of mortality. Approximately five billion patients are afflicted with hypertension globally. ${ }^{1}$ In the United States of America, with a $24 \%$ growth rate and $\$ 3.2$ trillion of the total spending on healthcare, $40 \%$ of hypertensive individuals (50 million people) remain untreated. ${ }^{3}$ Likewise, in the Mediterranean region, $26 \%$ of the total population is affected by hypertension. ${ }^{4}$ In developing countries, such as within the African region, hypertension has become concerning, with Ethiopia reporting an overall prevalence of $19.6 \%{ }^{5}$

Several influencing factors potentiate hypertension among individuals, such as nutritional transitions, lack of healthcare awareness, low economic status, inadequate healthcare systems, and adoption of increasingly sedentary lifestyles, which are factors more prevalent in the low- and middle-income countries (LMIC). ${ }^{6}$ Negligence in treatment and care of hypertension may result in cardiac, renal, and visual disorders. Hypertension is preventable, with a $10 \mathrm{~mm} \mathrm{Hg}$ reduction in systolic blood pressure (BP) resulting in a $32 \%$ reduction in stroke risk and $14 \%$ relative reduction in ischemic heart disease risk. ${ }^{7}$

To better control hypertension and prevent coronary vascular disease, experts have stressed the implementation of interventions such as salt restriction, body weight reduction, stress management, exercise, and alcohol intake reduction. Although national prevalence ratios differ depending upon the economy, social behaviors, and the population's level of healthcare awareness, hypertension has become a prevailing global medical condition. ${ }^{9}$

In Pakistan, the prevalence of hypertension is underreported due to the dearth of large population-based surveys. A cross-sectional survey ${ }^{9}$ reported an estimated prevalence of hypertension to be $26 \%$ in Pakistan, with a dominance of patients residing in the urban setting. In addition, Prospective Rural Urban Epidemiology (PURE) study ${ }^{8}$ found the combined prevalence of hypertension in Pakistan, Bangladesh and India hovering around $40 \%$.

To mitigate and prevent hypertension-related complications, self-care behavior adaptation is the most important and vital activity in ultimately reducing the risk factors associated with hypertension. The self-care regime consists of lifestyle modification, dietary changes, and adherence to medication. The lifestyle modification requires changes in daily routines involving proper diet, alcohol and smoking abstinence, exercise, and proper sleep. ${ }^{10}$ Past studies have shown effectiveness of self-care behaviors in reduction of BP and associated complications, plus escalation of the efficacy of medication and overall health regimes. ${ }^{4,5,30,36}$ Given the potential substantial benefits of self-care, novel approaches, and interventions are required to enable a robust and responsive system that can assist in promoting self-care behavior among individuals with hypertension.

Although existing pharmacologic therapies are effective, hypertension control over time is insufficient with medication therapy alone. ${ }^{11}$ The main purpose of the selfcare treatment and guidelines is to assist patient in BP control through sustainable non-pharmacological treatment. Various contributing factors may hinder hypertension control, but, most significantly, non-adherence towards self-care behaviors or lack of situational awareness respecting their condition will ultimately result in premature mortality and/or adverse health outcomes. Although over the last decade several medically driven hypertension treatment and management guidelines have been introduced, most have been rejected, or poorly adhered to, by hypertension-affected individuals. ${ }^{12}$ Nonadherence arises due to sub-factors such as lack of knowledge, understanding of healthcare interventions, and resources, as well as rigid mentality due to dominant of socio-cultural beliefs. ${ }^{13}$ The rejection and lack of uptake of self-care behaviors has contributed to early deaths, unhealthy lifestyles, chronic heart disease, and renal failure. Patients with risk factors for hypertension tend to avoid the beneficial self-care treatment primarily because of lack of awareness which, ultimately, results in lifethreatening or life-altering medical conditions or often death.

The PURE study reports a lack of quality data relating to cardiovascular risk factors and diseases from South Asia, a region that experienced a $73 \%$ increase in healthy life years lost due to ischemic heart disease between 1990 and 2010. These studies have allowed a better understanding of cardiovascular disease risk factors indigenous to the South Asian population along with conventional risk factors. The study recommends that culturally sensitive interventions geared towards treating risk factors identified in these studies are needed to fully realize the true potential of these epidemiologic studies. ${ }^{8}$

In contrast with traditional patient education, which focuses mainly on defining problems, self-management programs focus on developing problem-solving skills to help patients make decisions, take appropriate actions, and modify these actions under different circumstances. ${ }^{14}$ Self- 
management programs were found to decrease systolic BP by $5 \mathrm{mmHg}$ and diastolic BP by $4.3 \mathrm{mmHg}^{15}$ and increase cost-effectiveness. $^{16}$

The aim of this paper is to report the level of self-care and its predictors among hypertensive individuals in Pakistan. Secondly, the study identifies the foundations and specifications required to design an intervention to realize maximum efficiency and optimal outcomes related to hypertension control through a self-care approach.

\section{Methods}

This paper reports the results of a cross-sectional survey of a parent sequential mixed-method (QUANTI-quali) study designed to assess self-care behaviors and influencing factors among Pakistani hypertensive patients. The first phase of the study was a cross-sectional survey followed by qualitative indepth interviews, while the second phase included developing and feasibility testing of an intervention.

The study was conducted at two tertiary care hospitals of Karachi. Both hospitals were similar with respect to cardiac services and provided care across the spectrum of the socio-economically and ethnically diverse population of Karachi. The true prevalence of hypertension self-care among population of Karachi is unknown. Therefore, it was assumed to be 0.5 with an alpha set at $5 \%$ and within $5 \%$ of bound of error of estimation, the sample of 385 patients was required for the cross-sectional phase of the study. The sample was divided between the two settings on a 40:60 ratio based on the volume of patient influx in each hospital. Patients were recruited from the outpatient clinics between March and November 2019 based on the following criteria: adult (aged $\geq 18$ ), a diagnosis of primary hypertension for at least six months with prescribed antihypertensive medications to ensure documented hypertension, resident of Karachi, and ability to give informed consent. Patients identified with language barrier (inability to read and understand Urdu), documented malignancy, and physical or cognitive disability were excluded from the study.

Ethical approvals from the Ethics Review Committee and the Institutional Review Board of the respective settings were obtained prior to data collection (4712-SONERC-17 and IRD_IRB_2017_06_008). Information about the study and key steps of the protocol were shared with the physicians and clinic staff, including the nurses and unit receptionists, in the cardiology out-patient clinics of selected hospitals. The PI accessed the out-patient clinics one day before the data collection and screened patients scheduled for next day against the inclusion/exclusion criteria from the medical records. While waiting for their clinical appointments, eligible patients were approached by the PI who explained the purpose, procedure, and time of data collection. Those who volunteered to participate and gave a written consent were informed that confidentiality would be maintained by using a unique study ID. They were assured that refusal to participate would not affect their treatment plan at the hospital.

A self-developed checklist for the purpose of the study captured information on participants' demographic and socio-economic variables. The checklist assessed the participants' knowledge on the definition, causes and complications of hypertension. Self-care behaviors of the hypertensive patients were assessed using the H-SCALE developed by Warren-Findlow and Seymour ${ }^{17}$ to test the prevalence rates of hypertension self-care activities amongst African Americans. The H-SCALE assesses selfcare practices as outlined in the Seventh Report of the Joint National Committee on Prevention, Detection, Evaluation and Treatment of High Blood Pressure (JNC 8). It recommends 6 self-care behaviors: (1) adhering to medication regimens; (2) engaging in physical activity; (3) following a healthy, low-salt and low-fat diet similar to the Dietary Approaches to Stop Hypertension (DASH) diet; (4) maintaining a healthy weight; (5) reducing alcohol intake; and (6) avoiding tobacco.

The tool was translated into Urdu by two bilingual experts who were proficient in the source (English) as well as the target (Urdu) language. The finalized Urdu version was sent to four experts for content validation who rated the relevance and clarity of the tool on a Likert scale (1-4) and provided suggestions for the items which were rated $\leq 2$. The overall content validity index (CVI) for the H-SCALE tool was calculated to be 0.899 for clarity and 0.951 for relevance. Although the tool is typically a self-administered questionnaire, due to the low literacy rate in Pakistan data, was collected via a structured interviewing technique conducted by the PI and a trained data collector. The data collection approach was piloted with $10 \%$ of the sample size (ie, 40 patients) who were excluded from the final analysis. Discrepancies in the way of asking questions amongst the data collectors were resolved by mutual discussion.

The data collection forms were checked for clarity, accuracy, and completeness at the time of data collection. Each data form was dual entered and then analyzed with the Statistical Package for Social Sciences (SPSS ${ }^{\mathrm{TM}}$ ) Version 20.0. 
The dependent variable was the dichotomized total score of the constructs of the H-SCALE while the sociodemographic characteristics of the participants (ie, age, gender, education, marital status, living situation, and chronicity of the disease) were taken as the independent variables influencing self-care. Means and standard deviations or medians and inter-quartile ranges (IQR) were computed for continuous variables depending upon the distribution of the variables, whereas frequencies and percentages were calculated for categorical variables. Logistic regression was applied to determine associations between the dependent variable and independent variables.

\section{Results}

\section{Participants' Characteristics}

The median age of the study's 402 participants was 55 (ranging from 48 to 65 ) years. Approximately two thirds of study participants (66.17\%) were female, and most participants $(79.85 \%)$ were married and had some schooling $(67.16 \%)$. Approximately half $(54.73 \%)$ of the participants knew how to self-check their blood pressure at home. Of those knowing how to self-check blood pressure, $46.8 \%$ indicated checking only when symptoms appeared. Full participant characteristics are presented in Table 1.

\section{Knowledge Related to Hypertension}

Overall knowledge regarding hypertension was good (based on the pre-determined cut-off score) among approximately $4 \%$ of patients in the study. Across all subcategories, patients' awareness about complications of hypertension was relatively high $(17.91 \%)$ compared to knowledge of its definition (7.96\%), risk factors (11.69\%), and causes $(9.95 \%)$. Level of knowledge in each subcategory is presented in Table 2.

\section{Adherence to Self-Care}

The highest levels of adherence were observed for abstinence from alcohol (100\%), smoking cessation (83.33\%), and medication compliance $(71.89 \%)$, whereas the lowest levels of adherence were found for diet $(27.11 \%)$ and physical activity (24.88\%). Table 3 presents the level of adherence to each self-care behavior accessed via H-SCALE.
Table I Participants' Socio-Demographic Characteristics and the Level of Knowledge $(n=402)$

\begin{tabular}{|c|c|c|}
\hline Variable & $\begin{array}{l}\text { Frequency } \\
\text { (n) }\end{array}$ & $\begin{array}{l}\text { Percentage } \\
\text { (\%) }\end{array}$ \\
\hline \multicolumn{3}{|l|}{ Age (years) } \\
\hline $18-32$ & 42 & 10.44 \\
\hline $33-52$ & 83 & 20.65 \\
\hline $53-72$ & 201 & 50 \\
\hline 72 and above & 76 & 18.91 \\
\hline \multicolumn{3}{|l|}{ Gender } \\
\hline Male & 136 & 33.83 \\
\hline Female & 266 & 66.17 \\
\hline \multicolumn{3}{|l|}{ Education status } \\
\hline None & 132 & 32.84 \\
\hline School & 170 & 42.29 \\
\hline College & 38 & 09.45 \\
\hline University & 62 & 15.42 \\
\hline \multicolumn{3}{|l|}{ Marital status } \\
\hline Single & 15 & 03.73 \\
\hline Married & 321 & 79.85 \\
\hline Lost partner & 66 & 16.42 \\
\hline \multicolumn{3}{|l|}{ Family type } \\
\hline Alone & 16 & 03.98 \\
\hline Nuclear & 134 & 33.33 \\
\hline Extended & 252 & 62.69 \\
\hline \multicolumn{3}{|l|}{ Employment status } \\
\hline Unemployment & 48 & 11.94 \\
\hline Employment & 354 & 88.06 \\
\hline \multicolumn{3}{|l|}{ BP monitoring at home } \\
\hline Yes & 220 & 54.73 \\
\hline No & 182 & 45.27 \\
\hline \multicolumn{3}{|c|}{$\begin{array}{l}\text { Regularity of Self-Monitoring of } \\
\text { BP }\end{array}$} \\
\hline Regularly & 62 & 28.2 \\
\hline Rarely & 55 & 25.0 \\
\hline Subject to symptoms & 103 & 46.8 \\
\hline \multicolumn{3}{|l|}{ Overall knowledge of } \\
\hline \multicolumn{3}{|l|}{ Hypertension } \\
\hline Good & 18 & 04.47 \\
\hline Moderate & 170 & 42.28 \\
\hline Poor & 201 & 50 \\
\hline Not reported & 13 & 03.23 \\
\hline \multicolumn{3}{|l|}{$\mathrm{BP}$ monitoring at home } \\
\hline Yes & 220 & 54.73 \\
\hline No & 182 & 45.27 \\
\hline
\end{tabular}


Table 2 Knowledge Related to Hypertension

\begin{tabular}{|l|l|l|l|}
\hline Knowledge Category & Good Understanding n (\%) & Moderate Understanding n (\%) & *No Understanding n (\%) \\
\hline Definition of Hypertension & $32(7.96)$ & $235(58.46)$ & $135(33.58)$ \\
Risk Factors & $47(11.69)$ & $231(57.46)$ & $124(30.85)$ \\
Causes & $40(9.95)$ & $223(55.47)$ & $139(34.58)$ \\
Complications & $72(17.91)$ & $184(45.77)$ & $146(36.14)$ \\
\hline
\end{tabular}

Note: *For definition it was very limited understanding; rest was unknown.

Table 3 Adherence to Self-Care Behavior as Measured on H-SCALE

\begin{tabular}{|l|l|l|}
\hline Self-Care Behavior & Adherent $\mathbf{n}(\%)$ & Non-Adherent $\mathbf{n}(\%)$ \\
\hline Diet & $109(27.1 \mathrm{I})$ & $293(72.89)$ \\
Physical Activity & $100(27.88)$ & $302(75.12)$ \\
Medication Compliance & $289(71.89)$ & $113(28.11)$ \\
Smoking Cessation & $335(83.33)$ & $67(16.67)$ \\
Weight Monitoring & $219(54.48)$ & $183(45.52)$ \\
Abstinence from Alcohol & $402(100 \%)$ & $0(0 \%)$ \\
\hline
\end{tabular}

\section{Univariate Analysis}

At the univariate level, age, gender, education, and homebased blood pressure monitoring were found to be statistically significant in association with dietary self-care behavior. For medication adherence, age, gender, educational status, and blood pressure checking at home were significantly associated. With regard to physical activity, age, gender, marital status, and family type were statistically significant. Age, educational status, marital status, employment, and blood pressure checking at home were statistically significant at the univariate level for weight management amongst participants. For smoking cessation, age, gender, education, marital status, and homebased blood pressure monitoring showed statistically significant association.

\section{Multivariate Analysis}

At the multi-variable level, after controlling for age $(\mathrm{p}=0.001)$, education status $(\mathrm{p}=0.000)$, blood pressure checking at home $(\mathrm{p}=0.026)$, employment status $(\mathrm{p}=0.005)$, and knowledge of hypertension $(\mathrm{p}=0.019)$ were significantly associated with diet regimen. Similarly, gender (0.002) and blood pressure checking at home (0.038) remained key predictors of adherence to medication. For physical activity, age $(p=0.016)$ and gender $(p=0.000)$ retained statistical significance, while for weight management education status $(\mathrm{p}=$ $0.018)$ and blood pressure checking at home $(p=0.06)$ were statistically significantly associated. Age $(\mathrm{p}=0.008)$ and gen$\operatorname{der}(\mathrm{p}=0.015)$ were found to have significant association with adherence to non-smoking behavior in this study. The
Hosmer-Lemeshow goodness of fit statistic for each statistical model and values of Cronbach alpha for each construct are presented under each model in Tables 4 and 5.

\section{Discussion}

This study provides valuable insights about the knowledge, adherence to self-care, and its predictors among Pakistani adult patients with hypertension. A limited number of patients exhibited overall knowledge and awareness regarding hypertension. Across all subcategories, patients' awareness about complications of hypertension was relatively high compared to their knowledge of what hypertension is and how is it caused. The lack of awareness among the participants in the current study is consistent with findings of previous Pakistani studies, which also reported inadequate awareness about the risk factors related to NCDs affecting patients' adherence to lifestyle modifications. ${ }^{18,19}$ In contrast, studies in developed countries like $\mathrm{China}^{20}$ and the $\mathrm{USA}^{21}$ reported higher awareness and knowledge among patients who were living with hypertension, possibly attributable to differences in literacy rates of the population, as more than half of the participants in our study had no education which technically meant that they could not read and write their names. Despite widespread prevalence of hypertension, and its prediction to rise steeply, ${ }^{22}$ previous and current studies have shown that low patient awareness about the hypertension, its risks, and complications is ubiquitous.

Many studies ${ }^{23-25}$ report that the patients have poor to average knowledge regarding hypertension, despite of the range of sources of information available. Common factors impacting knowledge uptake with regards to Pakistan may be illiteracy and poverty. Being able to read and write privileges participants' access to education material available on the internet. It also gives the patient the confidence to discuss their learning needs with the health providers, whereas those who are not educated, tend to learn from their own experiences through a process of trial and error which may take longer than directly 
Table 4 Unadjusted Associations Between Different Socio-Demographic Characteristics and Self-Care Constructs on H-SCALE

\begin{tabular}{|c|c|c|c|c|c|}
\hline Variable & Medication Adherence & Diet & Physical Activity & Weight Control & Smoking \\
\hline \multicolumn{6}{|c|}{ Unadjusted OR $(95 \% \mathrm{CI})$} \\
\hline Age & $1.01(1.0-1.03)$ & $1.03(1.01-1.05)$ & $0.98(0.96-1.00)$ & $1.01(0.99-1.03)$ & $1.02(1.00-1.04)$ \\
\hline \multicolumn{6}{|l|}{ Gender } \\
\hline Male & $1.81(1.11-2.95)$ & $2.15(1.01-1.05)$ & $2.24(1.4 I-3.57)$ & Reference & Reference \\
\hline Female & Reference & Reference & Reference & $1.05(0.69-1.58)$ & $1.75(1.02-2.98)$ \\
\hline \multicolumn{6}{|l|}{ Education } \\
\hline School & $\mathrm{I} .44(0.88-2.35)$ & $1.50(0.85-2.66)$ & $1.18(0.68-2.03)$ & $1.81(1.14-2.86)$ & $0.89(0.47-1.68)$ \\
\hline College & 2.36 & $1.93(0.83-4.44)$ & I.5I (0.66-3.42) & $2.37(1.12-5.05)$ & $0.36(0.15-0.84)$ \\
\hline University & 1.83 & $5.75(2.93-11.27)$ & $1.76(0.89-3.47)$ & $1.50(0.8 \mathrm{I}-2.75)$ & $0.99(0.42-2.33)$ \\
\hline No Formal & Reference & Reference & Reference & Reference & Reference \\
\hline \multicolumn{6}{|l|}{ Marital Status } \\
\hline Single & Reference & I.0I (0.25-4.14) & Reference & Reference & Reference \\
\hline Married & $1.32(0.44-3.98)$ & $1.66(0.86-3.19)$ & $1.29(0.25-6.59)$ & $1.08(0.71-1.63)$ & $0.64(0.14-2.92)$ \\
\hline Lost Partner & $1.15(0.34-3.79)$ & Reference & $2.41(0.53-10.92)$ & $2.33(0.80-6.77)$ & $3.23(0.48-21.30$ \\
\hline \multicolumn{6}{|l|}{ Family Type } \\
\hline Alone & $0.71(0.32-3.34)$ & $1.62(0.56-4.63)$ & Reference & Reference & Reference \\
\hline Nuclear & $1.04(0.44-1.12)$ & $0.95(0.59-1.53)$ & $4.38(0.56-33.9)$ & $1.08(0038-3.08)$ & $1.60(0.47-5.43)$ \\
\hline Joint & Reference & Reference & $6.84(0.87-53.56)$ & $0.85(0.30-2.36)$ & $1.76(0.54-5.75)$ \\
\hline \multicolumn{6}{|l|}{ BP Checking } \\
\hline No & Reference & Reference & Reference & Reference & Reference \\
\hline Yes & $2.09(1.34-3.25)$ & $2.38(1.49-3.80)$ & $0.86(0.54-1.35)$ & $1.57(1.05-2.33)$ & $1.39(0.82-2.36)$ \\
\hline \multicolumn{6}{|l|}{ Employment } \\
\hline Employed & $0.64(0.30-1.33)$ & $1.99(0.90-4.4 I)$ & Reference & Reference & Reference \\
\hline Unemployed & Reference & Reference & I.I3 (0.57-2.25) & $0.68(0.36-1.27)$ & $0.68(0.27-0.39)$ \\
\hline \multicolumn{6}{|l|}{ Knowledge } \\
\hline Good & $0.92(0.52-1.28)$ & $2.56(0.93-7.02)$ & $1.37(0.46-4.05)$ & $2.33(0.80-6.77)$ & $0.98(0.26-3.58)$ \\
\hline Moderate & $0.82(0.52-1.28)$ & $2.08(1.30-3.33)$ & $\mathrm{I} .44(0.90-2.3 \mathrm{I})$ & $1.08(0.7 \mid-1.63)$ & $0.95(0.55-1.65)$ \\
\hline Poor & Reference & Reference & Reference & Reference & Reference \\
\hline
\end{tabular}

acquiring information from reliable sources. This finding aligns with the previous argument ${ }^{26,27}$ that self-care behavior can be learned experientially as well as taught to all irrespective of educational status. Other researchers have also pointed out that the existence of a communication gap between the patients and the healthcare providers regarding health education leads to a trend of low awareness regarding self-care practices. ${ }^{19,28}$ Therefore, there is a need in this context to develop stronger health education strategies which make basic knowledge of the disease accessible to all, regardless of demand and capacity. One such strategy, which may work in the context of LMIC, is to establish a routine of health education sessions embedded within the clinic visit to the hospital. These sessions should be delivered by nurses, as short, individualized, and targeted interventions on patient's needs in the language of preference of the patient. It should be built upon the existing level of knowledge of each patient based on their experience of living with hypertension.

As per the results of the study, abstinence from alcohol and smoking and adherence to medication were shown to be the highest adhered to self-care domains. All the patients reported abstinence from alcohol, which is likely due to the cultural and religious influence in which alcohol consumption is illegal and immoral. These findings align with previous studies which have shown that hypertensive patients are readily and actively involved in reduction of alcohol usage as part of self-care, ranging at a high level of adherence. $^{29-32}$ 
Table 5 Adjusted Associations Between Socio-Demographic Variables and Self-Care Behaviors on H-SCALE

\begin{tabular}{|c|c|c|c|c|c|}
\hline Variable & $\begin{array}{l}\text { Medication } \\
\text { Adherence }\end{array}$ & Diet & Physical Activity & Weight Control & Smoking \\
\hline \multicolumn{6}{|c|}{ Adjusted OR $(95 \% \mathrm{Cl})$} \\
\hline Age & & $1.03(1.01-1.05)$ & $0.97(0.95-0.99)$ & & $1.03(1.00-1.05)$ \\
\hline \multicolumn{6}{|l|}{ Gender } \\
\hline Male & $1.68(1.02-2.77)$ & & $2.47(1.53-3.98)$ & & Reference \\
\hline Female & Reference & & Reference & & $1.97(1.14-3.42)$ \\
\hline \multicolumn{6}{|l|}{ Education } \\
\hline School & & $1.14(0.61-2.13)$ & & $1.74(1.09-2.77)$ & \\
\hline College & & $1.03(0.4 \mathrm{I}-2.60)$ & & $2.12(0.99-4.57)$ & \\
\hline University & & $4.42(1.99-9.80)$ & & $1.36(0.73-2.52)$ & \\
\hline No Formal & & Reference & & Reference & \\
\hline \multicolumn{6}{|l|}{ BP Checking } \\
\hline No & Reference & Reference & & Reference & \\
\hline Yes & $2.0(1.28-3.12)$ & $1.79(1.07-3.0)$ & & $1.46(0.97-2.20)$ & \\
\hline \multicolumn{6}{|l|}{ Employment } \\
\hline Employed & & $4.09(1.51-11.05)$ & & & \\
\hline Unemployed & & Reference & & & \\
\hline \multicolumn{6}{|l|}{ Knowledge } \\
\hline Good & & $1.09(0.31-3.83)$ & & & \\
\hline Moderate & & $1.89(1.10-3.23)$ & & & \\
\hline Poor & & Reference & & & \\
\hline $\begin{array}{l}\text { Hosmer Lemeshow goodness of fit } \\
\text { Statistics }\end{array}$ & 0.10 & 0.73 & 0.72 & 0.252 & 0.015 \\
\hline Cronbach Alpha & 0.95 & 0.55 & 0.44 & 0.653 & 0.635 \\
\hline
\end{tabular}

Similarly, smoking is an under-reported phenomenon in Pakistan, particularly among females. ${ }^{33}$ In this study, two-thirds of the patients did not smoke, which may reflect that the majority of the participants were aware about the complications of hypertension, thus abstaining from smoking. In addition, smoking status was assessed through a self-reporting tool during a visit to the hospital that may have led to participants report "acceptable" behaviors, essentially resulting in potential reporting bias.

Medication adherence was seen in $72 \%$ of the participants amongst all socio-economic strata. Higher level of medication adherence has also been reported by previous studies ${ }^{19,27,30}$ conducted with cardiac patients where medication compliance was taken relatively seriously by the participants as compared to any other self-management behavior. Participants give importance to the prescription because they are personalized and directly written by the doctors whom they are answerable to on subsequent visits. ${ }^{27}$ The Pakistani population also has easy access to medications without prescriptions through readily available chemists. In addition, taking medications requires less effort compared to the other constructs. Moreover, the dominant culture constructs medications as the panacea to all health problems. ${ }^{34}$

Dietary practices and level of physical activity are the domains that require attention in the population studied. Diet is an essential part of self-care behavior to regulate hypertension with recommendation of a diet that is low in sodium, cholesterol, and saturated fats. ${ }^{9}$ Similar to the previous findings, ${ }^{4}$ educated patients in this study showed better adherence with diet and weight management than those who were less educated. Also, higher education was often associated with better economic status, which may increase patients' access to healthier food choices and physical activity programs that can potentially facilitate self-care behavior. 
Modification in the diet is a complex process that requires stepwise decision making. Abrupt changes in the traditional recipes can disrupt taste profiles of food, contributing to failure to adhere. In the Pakistani culture, food is replete with flavors and spices, and, importantly, sharing food is essential in socializing and maintaining relationships. It has a key role in linking family values, cultural beliefs, and individual behaviors, which makes it difficult for people to change their dietary patterns. An important aspect of their cultural beliefs includes consumption of the same food by all family members; hence, modification may be a deterrent for some people. ${ }^{35}$

Given the Pakistani context, health care workers are required to strive to advance patients' critical thinking and to develop an understanding of the suggested behaviors, thereby improving their self-care performance. Diet in this study is primarily driven by patients' age, education status, and employment. The Pakistani population require specific instructions from their health care providers, which are affordable, understandable, simple, and manageable for a primarily older and under-educated target group.

Physical activity is another important indicator of selfcare behavior that had low compliance in the current study. Physical activity is linked to control of high blood pressure, as it also leads to weight management, emotional control, and overall good health. This study revealed that an increase in age, male gender, married, and residing in a nuclear family setting were strong predictors of adherence to physical activity. In Pakistan, there is a lack of motivation in patients for formal physical activities due to poor accessibility to gyms, parks, and walking tracks. Regular exercise is not a part of the Pakistani way of life due to laziness, ignorance, lack of time, facilities, or presence of any other chronic diseases, such as arthritis or vertigo. $^{27}$

Men were more likely to be adherent to the activity regime, as culturally, they have greater access to the outdoor facilities compared to the women who are commonly confined to the house. Family systems also have the practice of the family spending the evenings together after a full day which will deter adherence. A number of studies $^{36-38}$ found that physical activity was one of the most difficult self-care behaviors to gain adherence, often due to misconceptions that it is comprised only of vigorous exercise. These findings highlight the need for increased physical activity in patients as well as putting forth a clearer message regarding the importance and contributions of physical activity.

\section{Strengths and Limitations}

The current study is adequately powered through a-priori sample size calculation to detect the true prevalence of self-care behaviors among patients with hypertension in Karachi Pakistan. It also captured the vast socio-economic and ethnic diversity among the study participants by enrolling participants from two large tertiary care hospitals of Karachi. However, given the cross-sectional survey methodology, causal inferences cannot be drawn between predictors and self-care behavior, hence results shall be construed cautiously. Also, the results of the study are based on a self-reported data that was collected by the data collector, which may be subjected to bias.

\section{Conclusion}

This study revealed that dietary modifications and regular activity patterns are self-care foci that require attention to enhance an overall healthy lifestyle to combat hypertension in the Pakistani population. Health care professionals are encouraged to offer context-based individualized strategies, which are economical, affordable, and understandable by the patients to enhance self-care practices for hypertension management.

\section{Abbreviations}

BP, Blood Pressure; CVD, Cardiovascular Diseases; NHS, National Health Security; SPSS, Statistical Package for Social Sciences; IQR, Inter-Quartile Range.

\section{Data Sharing Statement}

The data used and analyzed in the current study can be made available upon request to the corresponding author.

\section{Ethics Approval and Consent to Participate}

The study has been ethically approved by the ethics committee of the Aga Khan University Hospital and the Ethical Review Board of the Indus Hospital Karachi, Pakistan. The respondents were informed about the aim of the study. The respondents provided informed consent for the cross-sectional survey as well as the in-depth interviews. This study was conducted in accordance with the declaration of Helsinki. The confidentiality and anonymity of the data was also ensured.

\section{Acknowledgments}

AH: Aamir Hameed Khan, Section of Cardiology, Department of Medicine, Aga Khan University Hospital. 


\section{Author Contributions}

All authors made a significant contribution to the work reported, whether that is in the conception, study design, execution, acquisition of data, analysis and interpretation, or in all these areas; took part in drafting, revising or critically reviewing the article; gave final approval of the version to be published; have agreed on the journal to which the article has been submitted; and agree to be accountable for all aspects of the work.

\section{Funding}

All costs involved in the study were covered by the authors with no additional funding being received.

\section{Disclosure}

The authors report no conflicts of interest in this work.

\section{References}

1. Javadzade H, Larki A, Tahmasebi R, Reisi M. A theory-based self-care intervention with the application of health literacy strategies in patients with high blood pressure and limited health literacy: a protocol study. Int $J$ Hypertens. 2018;2018:4068538. doi:10.1155/2018/4068538

2. Mushtaq M, Najam N. Depression, anxiety, stress and demographic determinants of hypertension disease. Pak J Med Sci. 2014;30 (6):1293-1298. doi:10.12669/pjms.306.5433

3. Livet M, Haines ST, Curran GM, Seaton TL, Ward CS, Sorensen TD, Roth McClurg M. Implementation science to advance care delivery: a primer for pharmacists and other health professionals. Pharmacotherapy. 2018;38(5):490-502.

4. Niriayo YL, Ibrahim S, Kassa TD, et al. Practice and predictors of self-care behaviors among ambulatory patients with hypertension in Ethiopia. PLoS One. 2019;14(6):e0218947. doi:10.1371/journal. pone.0218947

5. Ademe S, Aga F, Gela D. Hypertension self-care practice and associated factors among patients in public health facilities of Dessie town, Ethiopia. BMC Health Serv Res. 2019;19(1):19-51. doi:10.1186/s12913-019-3880-0

6. Han H-R, Lee H, Commodore-Mensah Y, Kim M. Development and validation of the hypertension self-care profile: a practical tool to measure hypertension self-care. J Cardiovasc Nurs. 2014;29 (3):11-20. doi:10.1097/JCN.0b013e3182a3fd46

7. Thomopoulos C, Parati G, Zanchetti A. Effects of blood pressure lowering on outcome incidence in hypertension. 1. Overview meta-analyses, and meta-regression analyses of randomized trials J Hypertens. 2014;32:2285-2295.

8. Rehman H, Samad Z, Mishra SR, et al. Epidemiologic studies targeting primary cardiovascular disease prevention in South Asia. Indian Heart J. 2018;70(5):721-730. doi:10.1016/j.ihj.2018.01.029

9. Mubarik S, Malik SS, Mubarak R, Gilani M, Masood N. Hypertension associated risk factors in Pakistan: a multifactorial case-control study. Pak Med Assoc. 2019;69:1070-1073.

10. MacInnes J. Factors affecting self-care in heart failure: a literature review. Br J Card Nurs. 2008;3(7):293-299. doi:10.12968/bjca.2008.3.7.30495

11. Ding W, Li T, Su Q, Yuan M, Lin A. Integrating factors associated with hypertensive patients' self-management using structural equation modeling: a cross-sectional study in Guangdong, China. Patient Prefer Adherence. 2018;12:2169-2178. doi:10.2147/PPA.S180314
12. Basu S, Garg S, Sharma N, Singh MM. Improving the assessment of medication adherence: challenges and considerations with a focus on low-resource settings. Tzu Chi Med J. 2019;31(2):73-80. doi:10.4103/tcmj.tcmj_177_18

13. Dasgupta A, Sembiah S, Paul B, Ghosh A, Biswas B, Mallick N. Assessment of self-care practices among hypertensive patients: a clinic based study in rural area of Singur, West Bengal. Int J Community Med Public Health. 2017;5(1):262-267. doi:10.18203/2394-6040.ijcmph20175794

14. Bodenheimer T, Lorig K, Holman H, Grumbach K. Patient self-management of chronic disease in primary care. JAMA. 2002;288(19):2469-2475. doi:10.1001/jama.288.19.2469

15. Chodosh J, Morton SC, Mojica W, et al. Meta-analysis: chronic disease self-management programs for older adults. Ann Intern Med. 2005;143 (6):427-438. doi:10.7326/0003-4819-143-6-200509200-00007

16. Watson AJ, Singh K, Myint-U K, et al. Evaluating a web-based self-management program for employees with hypertension and prehypertension: a randomized clinical trial. Am Heart J. 2012;164 (4):625-631. doi:10.1016/j.ahj.2012.06.013

17. Warren-Findlow J, Seymour RB. Prevalence rates of hypertension self-care activities among African Americans. J Natl Med Assoc. 2011;103(6):503-512. doi:10.1016/S0027-9684(15)30365-5

18. Zafar SN, Gowani SA, Irani FA, Ishaq M. Awareness of the risk factors, presenting features and complications of hypertension amongst hypertensives and normotensives. Pak Med Assoc. 2008;58:711-715.

19. Bilal M, Haseeb A, Lashkerwala SS, et al. Knowledge, awareness and self-care practices of hypertension among cardiac hypertensive patients. Glob J Health Sci. 2016;8(9):9. doi:10.5539/gjhs.v8n9p159

20. Zhang W, Sun K, Yang Y, Zhang H, Hu FB, Hui R. Plasma uric acid and hypertension in a Chinese community: prospective study and metaanalysis. Clin Chem. 2009;55(11):2026-2034. doi:10.1373/ clinchem.2009.124891

21. Ong KL, Cheung BM, Man YB, Lau CP, Lam KS. Prevalence, awareness, treatment, and control of hypertension among United States adults 1999-2004. Hypertension. 2007;49(1):69-75. doi:10.1161/01.HYP.0000252676.46043.18

22. World Health Organization, Group ISoHW. World Health Organization (WHO)/International Society of Hypertension (ISH) statement on management of hypertension. J Hypertens. 2003;2003 (21):1983-1992.

23. Jankowska-Polańska B, Uchmanowicz I, Dudek K, Mazur G. Relationship between patients' knowledge and medication adherence among patients with hypertension. Patient Prefer Adherence. 2016;10:2437-2447. doi:10.2147/PPA.S117269

24. Akoko BM, Fon PN, Ngu RC, Ngu KB. Knowledge of hypertension and compliance with therapy among hypertensive patients in the Bamenda Health District of Cameroon: a cross-sectional study. Cardiol Ther. 2017;6(1):53-67. doi:10.1007/s40119-016-0079-x

25. Worku Kassahun C, Asasahegn A, Hagos D, et al. Knowledge on hypertension and self-care practice among adult hypertensive patients at University of Gondar comprehensive specialized hospital, Ethiopia, 2019. Int J Hypertens. 2020;2020:2020. doi:10.1155/2020/5649165

26. Strömberg A, Broström A, Dahlström U, Fridlund B. Factors influencing patient compliance with therapeutic regimens in chronic heart failure: a critical incident technique analysis. Heart Lung. 1999;28 (5):334-341. doi:10.1053/hl.1999.v28.a99538

27. Gowani A, Ahmed HI, Khalid W, et al. Facilitators and barriers to NCD prevention in Pakistanis-invincibility or inevitability: a qualitative research study. BMC Res Notes. 2016;9(1):282-290. doi:10.1186/s13104-016-2087-2

28. Bilal HSM, Akhtar U, Chul SW, Lee S, editors. Just in time intervention for personalized healthcare: behavior-context based intervention adaptation. 2020 International Conference on Information Networking (ICOIN). IEEE; 2020 Jan; Barcelona, Spain; p. 64-69. doi:10.1109/ICOIN48656.2020.9016632 
29. Kuhn L. The association between health literacy and diet adherence among primary care patients with hypertension. J Health Dispar Res Pract. 2014;7:109-127.

30. Warren-Findlow J, Seymour RB, Huber LRB. The association between self-efficacy and hypertension self-care activities among African American adults. J Community Health. 2012;37(1):15-24. doi:10.1007/s10900-011-9410-6

31. Uchmanowicz B, Chudiak A, Uchmanowicz I, Rosińczuk J, Froelicher ES. Factors influencing adherence to treatment in older adults with hypertension. Clin Interv Aging. 2018;13:2425-2441. doi:10.2147/CIA.S182881

32. Motlagh SFZ, Chaman R, Sadeghi E, Eslami AA. Self-care behaviors and related factors in hypertensive patients. Iran Red Crescent Med J. 2016;18:6.

33. Bush J, White M, Kai J, Rankin J, Bhopal R. Understanding influences on smoking in Bangladeshi and Pakistani adults: community based, qualitative study. BMJ. 2003;326(7396):962. doi:10.1136/ bmj.326.7396.962

34. Gowani A, Gul R, Dhakam S, Kurji N. Living with heart failure: Karachi exploratory study. Br J Card Nurs. 2017;12(12):586-592. doi:10.12968/bjca.2017.12.12.586
35. Barolia R, Petrucka P, Higginbottom GA, Khan FFS, Clark AM. Motivators and deterrents to diet change in low socio-economic Pakistani patients with cardiovascular disease. Glob Qual Nurs Res. 2019;6:6. doi:10.1177/2333393619883605

36. Alhalaiqa F, Al-Nawafleh A, Batiha A-M, Masa'deh R, Abd AlRazek A. A descriptive study of adherence to lifestyle modification factors among hypertensive patients. Turk J Med Sci. 2017;47:273-281. doi:10.3906/sag-1508-18

37. Girma F, Emishaw S, Alemseged F, Mekonnen A. Compliance with anti-hypertensive treatment and associated factors among hypertensive patients on follow-up in Jimma University specialized hospital, Jimma, south west Ethiopia: a quantitative cross-sectional study. Hypertension. 2014;3:174.

38. Gebrezgi MT, Trepka MJ, Kidane EA. Barriers to and facilitators of hypertension management in Asmara, Eritrea: patients' perspectives. J Health Popul Nutr. 2017;36(1):11. doi:10.1186/s41043-017-0090-4

\section{Publish your work in this journal}

The International Journal of General Medicine is an international, peer-reviewed open-access journal that focuses on general and internal medicine, pathogenesis, epidemiology, diagnosis, monitoring and treatment protocols. The journal is characterized by the rapid reporting of reviews, original research and clinical studies across all disease areas. The manuscript management system is completely online and includes a very quick and fair peer-review system, which is all easy to use. Visit http://www.dovepress.com/ testimonials.php to read real quotes from published authors. 